\title{
LDL-cholesterol and outcome prediction in patients with congestive heart failure
}

\section{Editorial}

Many large clinical studies advocated the use of statins in chronic heart failure (HF), assuming pleiotropic effects that are irrespective of cholesterol reduction. ${ }^{1-5}$ Treatment by statins is well established for primary and secondary prevention of coronary events. ${ }^{6}$ However, it became controversial in HF patients which as the data were mainly derived from patients with coronary heart disease. Sufficient data have been now provided to show that low cholesterol levels have a negative influence on the outcome in HF patients. ${ }^{4-5}$

There are still controversial data mainly based mainly on previous studies. $^{3-6}$ The findings of the BEST trial ${ }^{7}$ showed that cholesterol reduction by statins was independently associated with decrease in all-cause and cardiovascular mortality in patients with nonischemic dilated cardiomyopathy $(\mathrm{EF}<35$, NYHA-3-4). In contrast to these several other studies ${ }^{8,9-12}$ suggested that low total cholesterol levels are associated with increased mortality in patients with systolic nonischemic HF. According to Flammer et al., ${ }^{12}$ patients with HF who have intrinsically low cholesterol levels have a double risk of death up to 5years compared to patients with pharmacologically induced low cholesterol. Horwich et al., ${ }^{10}$ concluded that low serum total cholesterol is a detrimental factor in the unfavorable prognosis of HF patients. Somewhat surprisingly, the results of the CORONA study ${ }^{13}$ showed no survival or morbidity benefit in a group of patients with ischemic systolic HF who had been given low-dose rosuvastatin. This study concluded that myocardial infarction and stroke are relatively uncommon in systolic HF patients, and that rosuvastatin had no effect on the mortality rates from cardiovascular causes or sudden death.

Recently we reported a new data about the relationship between hypocholesterolemia and outcomes in elderly ${ }^{14}$ patients with advanced systolic and diastolic HF. Our 6-year longitudinal study results showed that low initial LDL cholesterol levels were a significant predictor of aggravated outcome in both ischemic and non-ischemic HF in an the elderly. The mortality rate in the patients with LDL $>115 \mathrm{mg} /$ dl) was reduced by $24 \%$ (in comparison to those with $\mathrm{LDL}<90$ ) despite the fact that the percent of patients with a NYHA class 3.54 was higher in this group. Even after adjusting for multiple known predictors of HF mortality, high LDL levels remained a significant novel independent predictor of improved survival. Neither low total cholesterol nor low triglycerides levels were significant in predicting mortality. The relationship between LDL levels and HF outcomes among the elderly, which was documented in the current study, seems to be contradictory to the findings in patients with coronary artery disease $^{3-4}$ and in patients enrolled in other HF studies ${ }^{2-7}$. Patients with ischemic and non-ischemic HF appear to have an opposite pattern ("reverse epidemiology"), with low levels being associated with worse prognosis independent of other prognostic factors. ${ }^{8,9}$ Rauchhaus et al., ${ }^{11}$ studied 414 patients with HF and found a $36 \%$ decreased risk of mortality with each millimole per liter increase in total cholesterol, independent of other risk factors.

There are several mechanisms by which high cholesterol levels may actually be protective in severely ill patients. Lipids and
Volume I Issue 2 - 2014

\author{
Gideon Charach,' Jacob George ${ }^{2}$ \\ 'Department of Internal Medicine "C", Tel Aviv University, Israel \\ ${ }^{2}$ Heart Center, Kaplan Medical Center, Hebrew University, Israel
}

Correspondence: Gideon Charach, Department of Internal Medicine "C", Tel Aviv Sourasky Medical Center, 6, Weitzman Street, Tel Aviv 6423906, Israel, Tel 9723 6973766,

Email drcharach@0I2.net.il

Received: May 19,2014 | Published: May 20, 2014

lipoproteins may play a protective role in HF by modulating the inflammation markers, such as $\mathrm{C}$-reactive protein, cytokines, oxidized LDL, tumor necrosis factor and interleukin $6 .{ }^{14,15}$ Cholesterol can bind endotoxin and liposaccharides of bacteria which are more common in severe HF and detoxify them, thus participating in the downregulation of inflammatory process and deactivating cytokines which contribute to myocyte damage. ${ }^{14,15}$ Treatment with statins was shown to reduce ubiquinone levels and thus could be potentially harmful. ${ }^{14}$ Thus, low LDL levels may cause HF patients to be vulnerable to inflammatory processes. However, a low LDL level may be a marker of disease severity. Advanced HF patients are characterized by a high catabolic state, increased metabolic demands and increased energy consumption. Our elderly patients with a range of LDL levels had the same normal albumin, creatinine, hemoglobin, and body mass index values which are established markers of the nutritional state. They also had normal levels of the inflammatory marker, C-reactive protein. All those factors indicate that as the main lipid predictor of atherosclerosis, the LDL cholesterol may be considered an independent predictor of mortality and not only the marker of the nutritional state. While the relation between cholesterol and atherosclerosis is indisputable, the questions of whether patients with HF need lipid-lowering treatment and what should be the optimal LDL levels remain unanswered. HF is a very late stage of most forms of cardiovascular disease when many cardiovascular alterations are irreversible. ${ }^{15}$

\section{Conclusion}

Low LDL cholesterol levels are associated with a reduced rate of survival in elderly patients with clinically controlled moderate and severe systolic and diastolic HF. Statins were independently and significantly associated with a higher mortality risk.

\section{Acknowledgments}

None.

\section{Conflicts of interest}

Authors declare that there is no conflict of interest.

\section{References}

1. Raina A, Pickering T, Shimbo D. Statin use in heart failure: a cause for concern? Am Heart J. 2006;152(1):39-49. 
2. Silva S, Lourenco P, Paulo C, et al. Statin-induced low cholesterol is not associated with poor outcome in chronic heart failure. J Cardiovasc Pharmacol Ther. 2012;17(3):284-290.

3. Song XJ, Yang CY, Liu B, et al. Atorvastatin inhibits myocardial cell apoptosis in a rat model with post-myocardial infarction heart failure by down regulating ER stress response. Int J Med Sci. 2011;8(7):564-572.

4. Khush KK, Waters DD. Effects of statin therapy on the development and progression of heart failure: mechanisms and clinical trials. J Card Fail. 2006;12(8):664-674.

5. Sakatani T, Shirayama T, Suzaki Y, et al. The association between cholesterol and mortality in heart failure. Comparison between patients with and without coronary artery disease. Int Heart J. 2005;46(4):619629.

6. Ford I, Murray H, Packard CJ, et al. Long-term follow-up of the West of Scotland Coronary Prevention Study. NEngl J Med. 2007;357(15):14771486 .

7. Domanski M, Coady S, Fleg J, et al. Effect of statin therapy on survival in patients with nonischemic dilated cardiomyopathy (from the Betablocker Evaluation of Survival Trial [BEST]). Am J Cardio. 2007;1 99(10):1448-1450.

8. Afsarmanesh N, Horwich TB, Fonarow GC.Total cholesterol levels and mortality risk in nonischemic systolic heart failure. Am Heart J. 2006;152(6):1077-1083.
9. Christ M, Klima T, Grimm W, et al. Prognostic significance of serum cholesterol levels in patients with idiopathic dilated cardiomyopathy. Eur Heart J. 2006;27(6):691-699.

10. Horwich TB, Hamilton MA, Maclellan WR, et al. Low serum cholesterol is associated with marked increase in mortality in advanced heart failure. J Card Fail. 2002;8(4):216-224.

11. Rauchhaus M, Clark AL, Doehner W, et al. The relationship between cholesterol and survival in patients with chronic heart failure. J Am Coll Cardiol. 2003;42(11): 1993-1940.

12. Flammer AJ, Sudano I, Wolfrum M, et al. Cardiovascular effects of flavanol-rich chocolate in patients with heart failure. Eur Heart J. 2011;33(17):2172-2180.

13. Kjekshus J, Apetrei E, Barrios V, et al. Rosuvastatin in older patients with systolic heart failure. $N$ Engl J Med. 2007;357(22):2248-2261.

14. Charach G, Rabinovich A, Ori A, et al. Low levels of low-density lipoprotein cholesterol: a negative predictor of survival in elderly patients with advanced heart failure. Cardiology. 2013;127(1):45-50.

15. Harris HW, Grunfeld C, Feingold KR, et al. Human very low density lipoproteins and chylomicrons can protect against endotoxin-induced death in mice. J Clin Invest. 1990;86(3):696-702. 